\title{
VALIDACIÓN DEL CUESTIONARIO DE ACTITUDES ANTE LAS INTERVENCIONES ASISTIDAS POR PERROS (CAINTAP) EN ESTUDIANTES UNIVERSITARIOS DEL SUR DE ESPAÑA: BENEFICIOS Y TEMORES PERCIBIDOS
}

\author{
Validation of the Questionnaire on Attitudes Towards Dog Assisted \\ Interventions (CAINTAP) among Southern Spanish college students: \\ perceived benefits and fears
}

\author{
Dr. Javier López-Cepero Borrego ${ }^{1,2}$, D. $^{a}$ María A. Perea-Mediavilla ${ }^{2,3}$, \\ D. ArCadio Tejada Roldán ${ }^{2}$, Dr. José Luis SARASOla-SÁnCHEZ-SERRANO ${ }^{3}$
}

\begin{abstract}
Resumen
Introducción: Las intervenciones asistidas por animales (IAA) cuentan con amplia tradición en varios países occidentales, donde diversas revisiones y meta-análisis han demostrado sus ventajas. No obstante, poco se sabe de las expectativas que los y las hispanohablantes mantienen al respecto. El presente estudio aporta una herramienta de evaluación sobre las actitudes hacia IAA. Material y métodos: un total de 474 estudiantes universitarios con edades de $X=22,7$ años $(d t=5,61)$, en su mayoría mujeres ( $80 \%)$, tomaron parte del estudio. El equipo de investigación desarrolló un instrumento de evaluación denominado Cuestionario sobre Actitudes hacia las Intervenciones Asistidas por Perros (CAINTAP). Resultados: el análisis factorial exploratorio retuvo 20 ítems en dos factores, etiquetados como Actitudes Positivas ( $\mathrm{N}=11)$ y Actitudes Negativas $(\mathrm{N}=9)$, ambos con alphas $>0,87$. El alumnado refirió mantener buenas expectativas hacia las IAA, mientras que los temores con mayor presencia correspondieron a comportamientos fácilmente modificables mediante adiestramiento. Discusión: este estudio aporta datos psicométricos del primer instrumento de evaluación sobre IAA en español, herramienta de utilidad para anticipar dificultades en centros en los que se desee llevar a cabo programas de intervención con perros.
\end{abstract}

Palabras clave: Intervenciones asistidas por animales, terapia asistida por animales, actividades asistidas por animales, estudiantes universitarios.

\begin{abstract}
Introduction: animal-assisted interventions (AAI) have a long tradition in some western countries, with many reviews and meta-analyses demonstrating their benefits, but little is known about expectations held by Spanish-speakers. This study develops an assessment tool, and provides empirical data on perceived opportunities and dangers. Methods: A total of $474 \mathrm{col}-$ lege students (aged $X=22.7$; $S D=5.61$ years), mostly women $(80 \%)$, took part in the study. Researchers developed the Questionnaire on Attitudes Towards Dogs Assisted Interventions (CAINTAP), which was then validated. Results: Exploratory factor analysis revealed a two-factor structure which retained 20 items, scales being coined Positive Attitudes $(\mathrm{N}=11)$ and Negative Attitudes $(\mathrm{N}=9)$, both with alphas greater than 0.87 . The students demonstrated good expectations for IAA, while the most frequent fears were related to easily modifiable behaviors. Discussion: This study provides psychometric data of the first questionnaire on IAA developed in Spanish, a useful tool that might help to anticipate difficulties regarding intervention with dogs.
\end{abstract}

Keywords: Animal-assisted interventions, animalassisted therapy, animal-assisted activities, college students.

1. Departamento de Personalidad, Evaluación y Tratamientos Psicológicos. Facultad de Psicología, Universidad de Sevilla. C/ Camilo José Cela, s/n. CP 41018, Sevilla. Teléfono: +34 954557801. E-mail: jalocebo@us.es. E-mails coautores: D. ${ }^{a}$ María A. Perea Mediavilla: manpermed@gmail. com, D. Arcadio Tejada Roldán: infointap@gmail.com, Dr. José Luis Sarasola-Sánchez-Serrano: jlsarsan@us.es.

2. INTAP.-Asociación Nacional de Intervenciones Asistidas por Perros y otros animales.

3. Universidad Pablo de Olavide. 


\section{Introducción}

Las intervenciones asistidas (IAA) se caracterizan por la introducción de animales como complemento a diversas disciplinas sociosanitarias y educativas (Hart, 2010). Estas intervenciones están recibiendo una atención creciente a nivel internacional, como demuestra el auge en el número de publicaciones científicas (López-Cepero et al., 2014) y la inclusión de descriptores específicos dentro de los Tesauros de bases de datos internacionales (como PsycINFO, que incluyó Animal-Assisted Therapy a mediados de 2013).

Aunque los animales han sido introducidos como facilitadores del cambio en diversos ámbitos desde el siglo XVIII (Serpell, 2010), la literatura recoge una amplia amalgama de términos y conceptos referentes a las IAA (Kruger y Serpell, 2010) que son utilizados de manera inconsistente (López-Cepero et al., 2012). No obstante, la propuesta que mayor arraigo ha conseguido hasta el momento entre las personas dedicadas a las IAA es la aportada por Pet Partners, entidad estadounidense anteriormente conocida como Delta Society, que distingue las actividades asistidas (encuentros puntuales que incluyen un animal, sin objetivos específicos más allá del propio ocio, y entre los cuales se contempla la educación asistida) y las terapias asistidas (que cuentan con diseño, programación y evaluación del cambio asimilables a cualquier otra terapia) (Pet Partners, sin fecha.) De todos los animales domésticos y de granja utilizados en las IAA, el perro es el que mayor presencia tiene en la literatura científica (Marino, 2012; Nimer y Lundhal, 2007; Perkins et al., 2008).

La interacción humano-animal ha sido empíricamente asociada a diversos efectos salutógenos, derivados tanto de la convivencia con el animal como mascota como de programas de intervención asistida (terapia, actividades, educación...). Entre los beneficios de la convivencia, por ejemplo, Virués-Ortega y Buela-Casal (2006) destacan la menor tasa cardiaca y mayor resistencia al estrés agudo de las personas que cuentan con mascotas, que podrían ser explicadas bien por la percepción de ser compañeros que no juzgan ni evalúan a sus dueños, bien por asociación condicionada con situaciones placenteras; en la misma línea, Barker y Wolen (2008) destacan la mayor tasa de supervivencia y adherencia a la rehabilitación en pacientes cardiópatas y la reducción de sintomatología depresiva, entre otros; por su parte Beetz, Uvnäs-Moberg, Julius y Kotrschal (2012) identifican la segregación de oxitocina como mediadora de los beneficios fisiológicos, sociales y de calidad de vida derivados de la IHA.

En cuanto a los beneficios obtenidos a través de programas de intervención asistida, la literatura ofrece diversos meta-análisis que recopilan resultados derivados de programas implementados en distintos colectivos. Nimer 
y col. (2007) analizaron 49 intervenciones de terapia asistida, encontrando evidencias de efectividad moderada en cuatro áreas de trabajo (trastornos del espectro autista, trastornos de conducta, bienestar emocional y problemas médicos); por su parte, Halm (2008) llevó a cabo un análisis centrado en intervenciones en ámbito hospitalario, encontrando hasta diez estudios que fueron clasificados de adecuados hasta totalmente recomendables por sus efectos positivos; en un tercer meta-análisis, Souter y Miller (2007), encontraron una relación significativa entre la participación en intervenciones asistidas y la mejora en pacientes diagnosticados de depresión mayor, diferencias que alcanzaron un tamaño de efecto medio en cinco estudios desarrollados con muestras estadounidenses. Revisiones bibliográficas como las realizadas por Filan y Llewellyn-Jones (2006), Perkins et al. (2008) o Steed y Smith (2002) también han destacado diversas ventajas aportadas por las intervenciones asistidas en personas mayores o con demencia. Por otra parte, Marino (2012) señala que un alto porcentaje de los estudios que ofrecen datos sobre la efectividad de las IAA incluyen debilidades metodológicas que pueden restar confiabilidad a sus conclusiones, si bien destaca que aquellos que sí incluyeron un control de los posibles sesgos (tales que el efecto placebo, efecto recencia, confusión conceptual, etc.) han encontrado efectos positivos de tamaño moderado derivados de las IAA, conclusión que coincide con lo apuntado por Nimer y col. (2007) y Souter y col. (2007).

A pesar de las evidencias a favor del uso de las IAA en distintos contextos, la literatura describe la ausencia de formación reglada durante los estudios de grado de disciplinas como Psicología, Medicina o Trabajo Social (Berget, Grepperud, Aasland y Braastad, 2013; Black, Chur-Hansen y Winefield, 2011; Risley-Curtiss, 2010), elemento que dificulta su inclusión durante el ejercicio profesional.

Varios estudios previos han analizado las actitudes de los profesionales hacia las intervenciones asistidas por animales. Ferrese, Forster, Kowalski y Wasilewski (1998; citado en Velde, Cipriani y Fisher, 2005) realizaron un análisis de las narraciones ofrecidas por terapeutas ocupacionales norteamericanos, destacando sus expectativas positivas en hasta siete áreas (aumento de motivación, beneficios físicos de la interacción, mejoras en el nivel de atención, mejoras del estado de ánimo, mejoras en la interacción social, ofrecer oportunidad de cuidar y crear un ambiente más similar al hogar). En una segunda experiencia, Black et al. (2011) llevaron a cabo entrevistas en profundidad a nueve psicólogos, concluyendo que las expectativas mezclaron tanto aspectos positivos (se esperaron mejoras en la relación terapéutica y en los resultados de la intervención) como negativos (temores ante la imprevisibilidad 
de los animales o la falta de apoyo de centros e instituciones) hacia las intervenciones asistidas.

Por otra parte, la literatura también incluye algunos estudios cuantitativos. Berget, Ekeberg y Braastad (2008) encontraron que dos tercios de una muestra de profesionales dedicados al cuidado psiquiátrico (médicos, psicólogos, enfermeros, etc.) consideraron que la inclusión de animales de granja podía mejorar la interacción entre terapeutas y pacientes, presentando mejores expectativas que hacia otras aproximaciones tradicionales de mayor arraigo. Berget y Grepperud (2011) concluyeron que entre un 55\% y un $87 \%$ de profesionales del ámbito psiquiátrico (sobre un total de 500 participantes) percibieron de alguna o mucha utilidad el uso de animales en el tratamiento de distintos trastornos mentales. En un trabajo posterior, Berget et al. (2013) cifraron en un $88 \%$ el porcentaje de que consideró necesario utilizar animales en las intervenciones con población psiquiátrica. Estos tres trabajos fueron realizados con muestras provenientes de Noruega. Por otra parte, en Australia, Moody, King y O'Rourke (2002) presentaron resultados derivados de la aplicación del Brisbane Animal Assisted Therapy Acceptability Test (BAATAT), único instrumento de evaluación de las actitudes ante las IAA localizado por nuestro equipo de investigación en la literatura, a los trabajadores de un ala pediátrica. Estos autores encontraron un alto nivel de acuerdo con las expectativas positivas antes de la implementación del programa de visitas, así como un descenso de las expectativas negativas después de la realización de dicha intervención.

En cuanto a las variables personales que pueden influir sobre la actitudes hacia las IAA, sólo el sexo del respondiente contó con apoyo empírico en la literatura previa, en la que son descritas expectativas más positivas en mujeres que en varones (Berget et al., 2008; Berget y col., 2011). Otras variables de interés estudiadas, como la experiencia con animales domésticos y/o de granja, la experiencia en intervenciones asistidas, el interés por participar en IAA o la profesión desempeñada no han mostrado una relación consistente en la literatura previa (Berget y col., 2011; López-Cepero, Perea-Mediavilla, Sarasola y Tejada, 2015; Moody et al., 2002; Perea-Mediavilla, López-Cepero, Tejada y Sarasola, 2014).

El presente trabajo se marca como objetivo ofrecer una evaluación cuantitativa de las actitudes hacia las IAA mantenidas por estudiantes universitarios de distintas Universidades Públicas de Andalucía Occidental. Para ello, se propone llevar a cabo la validación del Cuestionario de Actitudes ante las Intervenciones Asistidas por Perros (CAINTAP), ofreciendo datos acerca de su validez estructural, fiabilidad y validez concurrente. En un segundo 
momento, identificará las expectativas mantenidas por los estudiantes, ofreciendo información que pueda servir como guía para dar respuesta a los temores con mayor presencia entre los futuros profesionales.

\section{Método}

Participantes. El presente estudio contó con 474 estudiantes que se hallaron cursando estudios universitarios $(85,7 \%$ en grado o licenciatura; $14,3 \%$ en cursos de postgrado) de carácter presencial en las provincias de Huelva (19,0\% provenientes de la Universidad de Huelva) y Sevilla (34,7\% de la U. Pablo de Olavide y $46,3 \%$ de la U. de Sevilla). Un 50,8\% (N=251) de los participantes cursaron planes de estudios clasificados como Ciencias Sociales (Ciencias de la Educación, Trabajo Social, Educación Social, Sociología y Ciencias Políticas) y un 49,2\% ( $N=233$ ) lo hicieron en Ciencias de la Salud (Psicología y Enfermería). Por sexos, un 20,5\% refirió ser varón frente a un 79,5\% de mujeres (como punto de referencia, señalar que el Instituto Nacional de Estadística recogió en su último informe disponible, para el alumnado de nuevo ingreso del curso 2010-2011, que las mujeres representaron el 73,5\% en la rama de Ciencias de la Salud y el 60,6\% en Ciencias Sociales y Jurídicas en las tres universidades indicadas). La media de edad para el total de la muestra fue de 22,7 años $(\mathrm{dt}=5,61)$. Un 89,9\% de los participantes indicó no haber realizado ningún trabajo remunerado. Todos fueron mayores de edad y recibieron información sobre la voluntariedad de su participación. Una vez informados de los objetivos del estudio, se solicitó su asentimiento para participar, indicando datos de contacto del equipo investigador para atender dudas o preguntas que pudieran presentarse con posterioridad a la evaluación.

Instrumentos. La recogida de datos se realizó mediante la aplicación de tres formularios, presentados en un mismo documento. En primer lugar, fue incluida una hoja de variables sociodemográficas y educativas, en la que se solicitó información sobre el sexo, edad, estudios realizados y experiencia laboral del respondiente, a consignar en casilleros creados a tal efecto. En segundo lugar, se habilitó un apartado en el que describir la experiencia con animales de compañía (si existió y, en caso afirmativo, si fue positiva) y el interés por las aplicaciones de las IAA (si existió, si se poseyó formación o si se consideró de interés para la labor profesional, entre otras). Estos reactivos fueron respondidos marcando Sí/No en la hoja de respuestas.

El tercer formulario fue el Cuestionario de Actitudes ante las Intervenciones Asistidas por Perros (CAINTAP). Esta herramienta fue creada a través de una revisión de la literatura previa (ver Anderson, 2007; Wilson y Netting, 2012) 
y del Brisbane Animal Assisted Therapy Acceptance Test (BAATAT; Moody, King y O'Rourke, 2002). Dada la similitud entre varios ítems creados por el equipo de investigación para el presente trabajo y el BAATAT, se optó por incluir adaptaciones de 18 de los 21 elementos originales del instrumento de Moody et al. (2002), que fueron modificados para ser aplicables a diversos colectivos profesionales, y fueron incluidos 4 indicadores de nueva creación. Los 22 ítems resultantes evaluaron las expectativas de los participantes hacia cuestiones positivas (la intervención asistida beneficiará a la imagen del centro, los perros distraerán a las personas de sus preocupaciones) y negativas (los perros van a ladrar en el centro, los perros pueden ser una molestia para las personas que no participen de la intervención). Estos fueron respondidos usando una escala de cinco niveles de acuerdo, con valores comprendidos entre 1 (muy en desacuerdo) y 5 (muy de acuerdo) (ver Anexo).

Procedimiento. Se solicitó la colaboración de 19 docentes e investigadores, siguiendo un criterio de oportunidad, para que facilitaran el acceso a sus clases durante las últimas dos semanas del curso 2012-2013. 15 accedieron a participar, proporcionando acceso a 21 clases impartidas en 13 títulos diferentes. Un miembro del equipo se desplazó a cada una de estas clases para explicar los objetivos del estudio, asegurar la comprensión de las instrucciones y administrar las copias impresas. Se hizo constar la voluntariedad de la participación y fueron proporcionados datos de contacto del equipo de investigación.

Dadas las similitudes entre varios de los ítems extraídos de la literatura previa y el BAATAT, se optó por partir del instrumento de Moody et al. (2002), a fin de obtener información comparable con el trabajo original. Se llevó a cabo la traducción al español y una adaptación de contenidos que permitió ser de aplicación tanto a profesionales como a estudiantes, con independencia tanto del tipo de IAA (el original era de aplicación sólo a programas de visitas) como del colectivo con que se trabaje (no sólo en el área pediátrica). Tres ítems fueron eliminados ante la imposibilidad de aplicarlos fuera del contexto pediátrico, siendo sustituidos por nuevos elementos aplicables a diversos contextos de intervención. Los 22 ítems resultantes fueron administrados a varios colaboradores del equipo investigador a fin de comprobar y corregir posibles dificultades de redacción.

En el apartado estadístico fueron realizados procedimientos descriptivos (frecuencia, medidas de tendencia central, medidas de dispersión y análisis de distribución), análisis factorial exploratorio (extracción de componentes principales con autovalores mayores de 1, rotación Varimax), análisis de fiabilidad (alpha de Cronbach $>0,700$ ) correlaciones bivariadas ( $r$ de Pearson) y 
comparación de medias (prueba T de Student para muestras independientes). Estos procedimientos se realizaron a través del software SPSS, versión 20, utilizando un valor de significación de $p<0,05$. También se calculó el tamaño de efecto (TE) de las diferencias de medias, obtenido a través del valor resultante de dividir el valor absoluto de la diferencia de las medias de cada grupo entre la desviación típica del total de la muestra $[|X \mathrm{i}-X \mathrm{j}| / \sigma]$, considerándose para su valoración las orientaciones propuestas por Cohen (1988): tamaño de efecto pequeño para valores comprendidos $\geq 0,20 \mathrm{y}<0,50$; efecto moderado para valores $\geq 0,50$ y $<0,80$; y efecto grande para valores $\geq 0,80$.

\section{Resultados (I). Validación del CAINTAP.}

Validez Estructural. El análisis factorial exploratorio contó con una adecuación muestral suficiente (KMO=0,910; Esfericidad de Bartlett $p<0,000)$, ofreciendo una solución rotada con cuatro grupos de ítems (ver tabla 1). En total, la varianza explicada fue ligeramente inferior al $59 \%$. Una vez estudiada la matriz de la solución factorial, el equipo de investigación decidió retener sólo los dos primeros componentes, dada la diferencia en la varianza explicada (los dos primeros explicaron en torno al triple de varianza que los dos últimos) y el bajo número de reactivos contenidos en los factores $3 .^{\circ}$ y $4 .^{\circ}$

Dos ítems (numerados como 5 y 22) obtuvieron una saturación superior a 0,300 en ambos factores, por lo que el equipo investigador los asignó a la escala en la que presentaron mayor valor (dado que el reactivo 5 saturó negativamente, su puntuación aparece invertida en todos los cálculos realizados desde este punto en adelante). En total, 20 de los 22 ítems fueron retenidos en esta solución de dos factores, que fueron etiquetados como actitudes positivas hacia las IAA (factor 1) y actitudes negativas hacia las IAA (factor 2), respectivamente. La correlación entre ambos fue de $r=-0,468\left({ }^{* * *} p<0,001\right)$.

Fiabilidad. El índice alpha de Cronbach obtenido fue de 0,879 para la escala de Actitudes Positivas hacia las IAA (una vez invertido el valor del ítem 5) y de 0,884 para el factor de Actitudes Negativas hacia las IAA. Ninguno de estos valores mejoró por la exclusión de ítems. El valor de alpha para el total de 20 ítems fue de 0,658.

Validez concurrente. El análisis de la distribución de los dos factores identificados mostró un ajuste pobre a la curva normal en ambos casos, con valores $p<0,01$ o inferiores en las pruebas de Kolmogorov-Smirnov y Shapiro-Wilks, lo que justificó el uso de pruebas resistentes a esta situación en la comparación de medias obtenidas en el CAINTAP. 
Tabla 1. Solución factorial rotada (saturaciones $>0,300$ ) y varianza explicada. Entre paréntesis, valores de saturación de los ítems eliminados de cada escala por el equipo investigador

\begin{tabular}{|l|c|c|c|c|c|c|}
\hline \multirow{2}{*}{ Ítem (extracto) } & \multicolumn{3}{|c|}{ Factor } & \multirow{2}{*}{ X } & \multicolumn{1}{c|}{$\mathrm{dt}$} \\
\cline { 2 - 6 } & 1 & 2 & 3 & 4 & & \\
\hline 19. Actividad valiosa &, 827 & & & & 4,10 &, 937 \\
\hline 14. Residencia feliz &, 811 & & & & 4,13 &, 859 \\
\hline 16. Sitio más interesante &, 760 & & & & 4,07 &, 905 \\
\hline 21. Interés participantes &, 732 & & & & 4,23 &, 788 \\
\hline 18. Relax profesionales &, 680 & & & & 3,68 & 1,099 \\
\hline 10. Relax participantes &, 677 & & & & 4,11 &, 864 \\
\hline 13. Acuerdo profesionales &, 644 & & $(-, 335)$ & & 3,51 &, 994 \\
\hline 20. Familiares de acuerdo &, 620 & & $(-, 315)$ & & 3,34 &, 976 \\
\hline 4. Imagen del centro &, 616 & & & $(, 387)$ & 3,90 &, 940 \\
\hline 5. No es lugar adecuado* &,- 516 & $(, 389)$ & & & 2,32 & 1,194 \\
\hline 2. Menos carga de trabajo &, 406 & & & $(-, 342)$ & 2,46 & 1,087 \\
\hline 11. Pulgas/garrapatas & &, 863 & & & 2,26 & 1,221 \\
\hline 12. Infecciones & &, 856 & & & 2,19 & 1,156 \\
\hline 9. Morder & &, 821 & & & 2,24 & 1,159 \\
\hline 17. Arañar & &, 766 & & & 2,62 & 1,170 \\
\hline 15. Dañar instrumental & &, 718 & & & 2,72 & 1,184 \\
\hline 22. Molestia no participantes & $(-, 333)$ &, 469 & $(, 352)$ & & 3,02 & 1,141 \\
\hline 7. Alergias & & &, 766 & & 3,76 & 1,008 \\
\hline 6. Problemas respiratorios & &, 334 &, 721 & & 3,10 & 1,145 \\
\hline 1. Ladrar & &, 390 & &, 714 & 3,42 & 1,146 \\
\hline 3. Orinar/defecar &, 463 & &, 674 & 3,21 & 1,303 \\
\hline 8. Distraer de preocupaciones & & & &, 367 & 3,66 & 1,287 \\
\hline Varianza explicada & $23,96 \%$ & $20,03 \%$ & $7,61 \%$ & $7,30 \%$ & & \\
\hline
\end{tabular}

*El ítem 5 saturó de manera inversa en el factor 1.

El primer contraste realizado comparó las medias obtenidas en los dos factores propuestos para el CAINTAP según la variable sexo del respondiente. Las puntuaciones obtenidas por las mujeres participantes fueron descriptivamente superiores en la escala de actitudes positivas, mientras que las medias para el factor de actitudes negativas se distanciaron en menos de una 
Tabla 2. Actitudes ante las IAA en varones y mujeres. Se incluyen datos descriptivos, prueba de Levene para igualdad de varianzas y prueba T para igualdad de medias

\begin{tabular}{|l|l|r|c|c|c|c|c|c|c|c|}
\hline \multicolumn{2}{|c|}{ Sexo } & \multicolumn{4}{|c|}{ Descriptivos } & \multicolumn{2}{c|}{$\begin{array}{c}\text { Igualdad } \\
\text { var. }\end{array}$} & \multicolumn{3}{c|}{ Prueba T } \\
\cline { 3 - 12 } \multicolumn{2}{|c|}{} & \multicolumn{1}{|c|}{$\mathrm{N}$} & $X$ & $\mathrm{dt}$ & $\mathrm{e}_{\mathrm{t}}$ & $\mathrm{F}$ & $p$ & $t$ & $\mathrm{gl}$ & $p$ \\
\hline \multirow{2}{*}{ Act. Positiva } & Hombre & 93 & 40,56 & 6,83 & 0,71 &, 171 &, 679 & $-1,104$ & 457 &, 270 \\
\cline { 2 - 12 } & Mujer & 366 & 41,48 & 7,28 & 0,38 & & & & & \\
\hline Act. Negativa & Hombre & 95 & 24,72 & 6,95 & 0,71 & 2,678 &, 102 &,- 110 & 461 &, 913 \\
\cline { 2 - 11 } & Mujer & 368 & 24,81 & 7,82 & 0,41 & & & & & \\
\hline
\end{tabular}

centésima de punto. Ninguna de estas diferencias alcanzó significatividad estadística en el contraste T de Student (tabla 2).

La segunda variable cualitativa seleccionada fue el interés por incluir programas de IAA en el contexto laboral del respondiente. De media, las personas que indicaron tener interés por esta modalidad de intervención mostraron actitudes más favorables ante las IAA. Estas diferencias alcanzaron un nivel de significación estadística de $p<0,001$ para ambas escalas del CAINTAP (tabla 3). Tomando como referencia la media y desviación típica halladas para ambos factores (Actitudes Positivas: $X=41,29 ; \mathrm{dt}=7,19$ / Actitudes Negativas: $X=24,79$; $\mathrm{dt}=7,64$ ), el tamaño de efecto para ambos contrastes fue grande (Cohen, 1988).

Tabla 3. Actitudes ante las IAA según interés mostrado por incluir programas de IAA en contexto laboral. Se incluyen datos descriptivos, prueba de Levene para igualdad de varianzas, prueba $\mathrm{T}$ para igualdad de medias y tamaño de efecto

\begin{tabular}{|c|c|c|c|c|c|c|c|c|c|c|c|}
\hline \multirow{2}{*}{\multicolumn{2}{|c|}{$\begin{array}{c}\text { ¿Interés en la } \\
\text { IAA? }\end{array}$}} & \multicolumn{4}{|c|}{ Descriptivos } & \multicolumn{2}{|c|}{$\begin{array}{l}\text { Igualdad } \\
\text { var. }\end{array}$} & \multicolumn{3}{|c|}{ Prueba T } & \multirow[t]{2}{*}{ TE } \\
\hline & & \multirow{2}{*}{$\frac{\mathrm{N}}{401}$} & \multirow{2}{*}{$\frac{X}{42,50}$} & \multirow{2}{*}{$\frac{\mathrm{dt}}{6,14}$} & \multirow{2}{*}{$\frac{e_{t}}{0,31}$} & \multirow{2}{*}{$\frac{F}{2,106}$} & \multirow{2}{*}{$\frac{p}{, 147}$} & \multirow{2}{*}{$\frac{t}{11,390}$} & \multirow{2}{*}{$\frac{\mathrm{gl}}{448}$} & \multirow{2}{*}{$\frac{p}{, 000 * * *}$} & \\
\hline Act. Positiva & Sí & & & & & & & & & & 1,52 \\
\hline & No & 49 & 31,57 & 7,81 & 1,12 & & & & & & \\
\hline \multirow{2}{*}{$\begin{array}{l}\text { A c t t } \\
\text { Negativa }\end{array}$} & Sí & 403 & 24,10 & 7,35 & 0,37 & ,036 & 849 & $-5,522$ & 452 &, $000 * * *$ & 0,80 \\
\hline & No & 51 & 30,18 & 7,79 & 1,09 & & & & & & \\
\hline
\end{tabular}

$* * * p<0,001$

Alternativas. Cuadernos de Trabajo Social, 22, 2015, pp. 123-138 - ISSN 1133-0473 DOI: 10.14198/ALTERN2015.22.07 
Tabla 4. Actitudes ante las IAA según si el respondiente ha convivido con mascotas. Se incluyen datos descriptivos, prueba de Levene para igualdad de varianzas, prueba $\mathrm{T}$ para igualdad de medias y tamaño de efecto

\begin{tabular}{|c|c|c|c|c|c|c|c|c|c|c|c|}
\hline \multirow{2}{*}{\multicolumn{2}{|c|}{ ¿Mascota? }} & \multicolumn{4}{|c|}{ Descriptivos } & \multicolumn{2}{|c|}{$\begin{array}{l}\text { Igualdad } \\
\text { var. }\end{array}$} & \multicolumn{3}{|c|}{ Prueba T } & \multirow{3}{*}{$\begin{array}{c}\text { TE } \\
0,51\end{array}$} \\
\hline & & \multirow{2}{*}{$\frac{\mathrm{N}}{401}$} & \multirow{2}{*}{\begin{tabular}{|c|}
$X$ \\
41,76
\end{tabular}} & \multirow{2}{*}{$\frac{\mathrm{dt}}{7,00}$} & \multirow{2}{*}{$\frac{e_{t}}{0,35}$} & \multirow{2}{*}{\begin{tabular}{c|}
$F$ \\
927
\end{tabular}} & \multirow{2}{*}{$\frac{p}{336}$} & \multirow{2}{*}{$\frac{t}{-3,704}$} & \multirow{2}{*}{$\frac{\mathrm{gl}}{457}$} & \multirow{2}{*}{$\frac{p}{, 000^{* * *}}$} & \\
\hline Act. Positiva & Sí & & & & & & & & & & \\
\hline & No & 58 & 38,07 & 7,70 & 1,01 & & & & & & \\
\hline \multirow[t]{2}{*}{ Act. Negativa } & Sí & 405 & 24,24 & 7,54 & 0,37 & ,151 & ,697 & 4,187 & 461 &, $000 * * *$ & 0,60 \\
\hline & No & 58 & 28,66 & 7,31 & 0,96 & & & & & & \\
\hline
\end{tabular}

${ }^{* * *} p<0,001$

En tercer lugar, fueron comparadas las actitudes de los respondientes que informaron haber convivido con mascotas en algún momento de la vida y quienes refirieron no haber tenido animales domésticos a cargo. Las personas con experiencia en la convivencia con animales no humanos mostraron actitudes más favorables ante las IAA, diferencias que alcanzaron significación estadística $(p<0,001)$ en las dos escalas del CAINTAP (tabla 4). El tamaño de efecto para ambos contrastes fue moderado (Cohen, 1988).

\section{Resultados (II). Expectativas más frecuentes en torno a las IAA.}

La media obtenida en el total de la muestra para la escala de actitudes positivas ante las IAA fue de 41,3 puntos, siendo estadísticamente superior al punto medio esperado por azar ( $p<0,001$; ver tabla 5$)$. Cinco ítems de esta escala obtuvieron puntuaciones medias superiores a 4 puntos, situándose entre los valores «de acuerdo»y «muy de acuerdo»: «la IAA resultará interesante para los posibles participantes», «la IAA hará del centro un lugar más feliz», «la IAA ayudará a los participantes a relajarse», «este programa será una actividad valiosa para el centro» $\mathrm{y}$ «el centro será un sitio más interesante para trabajar». Entre los diez ítems con medias más altas se encontraron ocho de este factor junto con los dos reactivos eliminados en el análisis factorial exploratorio.

En cuanto a la escala de actitudes negativas ante las IAA, destaca que la media encontrada para el total de la muestra fuera estadísticamente inferior al punto medio teórico ( $p<0,001$; ver tabla 5$)$. Fueron obtenidos datos descriptivos de los ítems de esta escala para el total de la muestra, de las personas que indicaron no tener interés en desarrollar IAA en el futuro, y de las personas que no han tenido nunca una mascota al cargo (ver tabla 6). Los tres grupos 
Tabla 5. Prueba T para las medias de los factores del CAINTAP con respecto al punto medio teórico, producto del número de ítems multiplicado por 3, el valor medio de la escala

\begin{tabular}{|l|c|c|c|c|c|c|c|c|}
\hline & $\mathbf{N}$ & $\begin{array}{c}\text { Valor } \\
\text { prueba }\end{array}$ & $\boldsymbol{X}$ & $\mathrm{dt}$ & $\mathrm{e}_{\mathrm{t}}$ & $\boldsymbol{t}$ & $\mathrm{gl}$ & $\boldsymbol{p}$ \\
\hline Act. Positivas & 459 & 33 & 41,29 & 7,193 &, 336 & 24,706 & 458 &, $000^{* * *}$ \\
\hline $\begin{array}{l}\text { Act. } \\
\text { Negativas }\end{array}$ & 463 & 27 & 24,79 & 7,644 &, 355 & $-6,213$ & 462 &, $000^{* * *}$ \\
\hline
\end{tabular}

Tabla 6. Medias y desviación tipo para los ítems del factor de actitudes negativas del CAINTAP en el total de la muestra y para grupos seleccionados.

\begin{tabular}{|l|c|c|c|c|c|c|}
\hline & \multicolumn{2}{|c|}{$\begin{array}{c}\text { Total muestra } \\
(\mathrm{N}=474)\end{array}$} & \multicolumn{2}{c|}{$\begin{array}{c}\text { No desean IAA } \\
(\mathrm{N}=52)\end{array}$} & \multicolumn{2}{c|}{$\begin{array}{c}\text { Sin mascota } \\
(\mathrm{N}=60)\end{array}$} \\
\hline \multicolumn{1}{|c|}{ Ítem (extracto) } & $X$ & $\mathrm{dt}$ & $X$ & $\mathrm{dt}$ & $X$ & $\mathrm{dt}$ \\
\hline 1. Ladrar & 3,42 & 1,15 & 3,88 & 1,17 & 3,63 & 1,19 \\
\hline 3. Orinar/defecar & 3,21 & 1,30 & 3,55 & 1,33 & 3,55 & 1,30 \\
\hline 6. Problemas respiratorios & 3,10 & 1,15 & 3,61 & 1,20 & 3,60 &, 98 \\
\hline 22. Molestia no participantes & 3,02 & 1,14 & 3,62 & 1,12 & 3,50 & 1,03 \\
\hline 15. Dañar instrumental & 2,72 & 1,18 & 3,40 & 1,14 & 3,13 & 1,13 \\
\hline 17. Arañar & 2,62 & 1,17 & 3,15 & 1,07 & 3,03 & 1,13 \\
\hline 11. Pulgas/garrapatas & 2,26 & 1,22 & 3,06 & 1,26 & 2,75 & 1,32 \\
\hline 9. Morder & 2,24 & 1,16 & 2,84 & 1,24 & 2,80 & 1,26 \\
\hline 12. Infecciones & 2,19 & 1,16 & 3,02 & 1,21 & 2,72 & 1,26 \\
\hline Total escala & 24,79 & 7,64 & 30,18 & 7,79 & 28,66 & 7,31 \\
\hline
\end{tabular}

coincidieron en apuntar como dificultades más probables: «los perros van a ladrar en el centro», "es probable que los perros orinen o defequen en el centro», "puede que los perros empeoren determinados problemas respiratorios» $\mathrm{y}$ «los perros pueden ser una molestia para las personas que no participen en la intervención».

\section{Discusión y conclusiones}

El presente trabajo muestra información de interés sobre actitudes hacia las intervenciones asistidas por animales, aportando datos psicométricos del primer instrumento disponible en español (CAINTAP) e identificando las 
principales virtudes y riesgos percibidos por futuros profesionales de diversos ámbitos sociosanitarios y educativos.

En cuanto a la validación del cuestionario, la solución rotada de cuatro factores guarda ciertos parecidos con la propuesta de Moody et al. (2002). Sin embargo, el cambio en los contenidos y número de ítems incluidos en el CAINTAP, el bajo número de elementos contenidos por las dos últimas escalas y las grandes diferencias en cuanto a varianza explicada justificó la elección de una nueva solución que contuvo sólo dos factores. Esta decisión permitió retener 20 de los 22 ítems propuestos con una varianza explicada del $44 \%$. Por otra parte, los valores de alpha superaron el valor de 0,850 en ambos casos, con un valor de 0,650 para el total del instrumento, siendo estos datos superiores a los descritos por Moody et al. (2002). Estos indicios psicométricos, en combinación con la mayor facilidad para interpretar los resultados obtenidos a través del instrumento, hacen preferible la estructura de dos factores a los cuatro que aparecen en la propuesta original.

El presente trabajo también ofrece los primeros resultados acerca de la relación entre actitudes hacia las IAA y otras variables de interés en muestra española. Con respecto al sexo del respondiente, se encontró que las actitudes hacia las IAA fueron estadísticamente similares entre varones y mujeres, no corroborándose el efecto que dicha variable ha mostrado en trabajos previos (Berget et al., 2008; Berget y col., 2011). Este resultado puede estar mediatizado por la baja proporción de varones en la muestra (en torno a un $20 \%$ del total), si bien también debe plantearse el efecto de las diferencias culturales entre muestras (noruega y española). Sí se han encontrado diferencias actitudinales al comparar medias entre grupos diferenciados por otras dos variables de interés: la utilidad percibida de las IAA para la labor profesional y la experiencia previa con mascotas. A pesar de la ausencia de antecedentes en la literatura, los resultados han apuntado en la dirección esperada (mejores actitudes entre personas que consideran las IAA como un recurso valioso frente a quienes no la consideran, y actitudes más positivas entre quienes han estado al cuidado de mascotas frente a quienes nunca han tenido ninguna), con tamaños de efecto de intermedios a grandes. Estos hallazgos suponen otro apoyo a la validez del CAINTAP.

Por otra parte, el presente trabajo ha permitido extraer conclusiones relevantes acerca de las expectativas que los estudiantes universitarios de diversas ramas y especialidades mantienen acerca de las IAA, si bien el método de muestreo seguido (no probabilístico e inferior al $1 \%$ de la población estudiantil de las universidades implicadas) hace necesario extremar la precaución de cara a generalizar las conclusiones alcanzadas. 
En primer lugar, los resultados destacan que las actitudes hacia este tipo de intervención son positivas. Este hallazgo resulta especialmente relevante si se contrapone al hecho de que no exista formación reglada sobre las IAA en los Estudios de Grado en España, lo que conlleva que los futuros profesionales carezcan en su mayoría de conocimientos basados en la evidencia sobre las posibilidades y limitaciones de las IAA. En segundo lugar, el presente trabajo ha cuantificado y destacado que las actitudes negativas más extendidas, tanto para el total de la muestra como para los grupos seleccionados, corresponden a elementos fácilmente controlables por el equipo de intervención (por ejemplo, temor a que los animales ladren u orinen/defequen en el centro). En este sentido, los resultados presentados pueden servir de guía a los profesionales del área a la hora de orientar la presentación de sus programas, haciendo especial hincapié en los aspectos que suscitan mayores recelos entre los profesionales de distintos ámbitos.

Por todo lo apuntado, el CAINTAP aparece como un recurso valioso para todos los profesionales que deseen evaluar las expectativas que los profesionales del centro mantienen acerca de los programas de IAA. Esta herramienta es capaz de discriminar entre personas que difieren en opiniones y experiencias teóricamente relevantes, al mismo tiempo que ha mostrado adecuados niveles de fiabilidad. Por todo lo expuesto, el CAINTAP se erige como una opción adecuada para evaluar actitudes ante las IAA en contextos de intervención e investigación, llenando el vacío existente de instrumentos validados en nuestro idioma, y aportando diversas ventajas: es aplicable a cualquier persona, ya sea estudiante o profesional; puede administrarse con independencia del colectivo con el que trabaje, superando así a herramientas validadas en otros idiomas; y ofrece información relevante sobre las expectativas hacia las IAA en un determinado contexto de intervención, posibilitando dar una respuesta adecuada a las expectativas (positivas y negativas) mantenidas mediante la presentación de una visión más real y ajustada a la evidencia de las posibilidades de las IAA.

\section{Bibliografía}

Anderson, D.C. (2007). Assessing the Human-Animal Bond. EE. UU: Purdue University Press.

BARKER, S.B. y WOLEN, A.R. (2008). The benefits of human-companion animal interaction: a review. Journal of Veterinaty Medical Education, 35, 487-495. DOI: $10.3138 /$ jvme.35.4.487

BeETZ, A., UvNÄS-MoberG, K, Julius, H. y KotrsChal, K. (2012). Psychosocial and psychophysiological effects of human-animal interactions: the possible role of oxitocin. Frontiers in Psychology, 3, 1-15. DOI: 10.3389/fpsyg.2012.00234 
Berget, B., Ekeberg, O. and BraAstad, B.O. (2008). Attitudes to animal-assisted therapy with farm animals among health staff and farmers. Journal of Psychiatric and Mental Health Nursing, 15, 576-581. DOI: 10.1371/journal. pone.0083993

Berget, B. and GrepPerud, S. (2011). Animal-assisted interventions for psychiatric patients: beliefs in treatment effects among practitioners. European Journal of Integrative Medicine, 3, e91-e96. DOI: 10.1016/j.eujim.2011.03.001

Berget, B., Grepperud, S., Aasland, O.G. and BraAstad, B.O. (2013). Animalassisted interventions and psychiatric disorders: knowledge and attitudes among general practitioners, psychiatrist, and psychologists. Society and Animals, 21, 284-293. DOI: 10.1163/15685306-12341244

Black. A.F. Chur-Hansen. A., y Winefield. H.R. (2011). Australian psychologists' knowledge of and attitudes towards animal-assisted therapy. Clinical Psychologist, 15, 69-77. DOI: 10.1111/j.1742-9552.2011.00026.x

Cohen, J. (1988). Statistical Power Analysis for the Behavioral Sciences. (2a ed). NJ, EEUU: Erlbaum

FILAN, S.L. y LLEWELlYN-JONES, R.H. (2006). Animal-assisted therapy for dementia: a review of the literature. International Psychogeriatric, 18, 597-611. DOI: $10.1017 /$ S1041610206003322

HALM. M.A. (2008). The healing power of the human-animal connection. American Journal of Critical Care. 17. 373-376.

HART, L.A. (2010). Possitive effects of animals for psychosocially vulnerable people: a turning point for delivery. En H.A. Fine (Ed.). Handbook of animalassisted therapy: Theoretical foundations and guidelines for practice $\left(3 .^{a} \mathrm{ed}\right.$.) (pp.59-84). U.S.A.: Academic Press.

KRUGER. K.A., \& SERPELl. J.A. (2010). Animal-assisted interventions in mental Elath: definitions and theoretical foundations. In H.A. Fine (Ed.). Handbook of animal-assisted therapy: Theoretical foundations and guidelines for practice (3. ${ }^{a}$ ed.) (pp.33-48). U.S.A.: Academic Press.

lópez-Cepero, J., Blanco, N., Perea-Mediavilla, M.A., Tejada, A., RodríGuezFranco, L. y Blanco, A. (2012). Una aproximación al estado actual de las intervenciones asistidas por animales en la literatura científica. Logros y retos de futuro. En R. Quevedo-Blasco y V.J. Quevedo-Blasco (eds.), Avances en Psicología Clínica (pp. 898-992). Granada: AEPC.

López-Cepero, J., Perea-Mediavilla, M.A., Sarasola, J.L. y Tejada, A. (2015). Influence of biographical variables and academic background on attitudes towards animal assisted interventions. Human-Animal Interaction Bulletin, 3, 10-18.

López-Cepero, J., Rodríguez-Franco, L., Perea-Mediavilla, M.A., Blanco, N., TejadA, A. y Blanco, A. (2014). Animal-assisted interventions: review of 
current status and future challenges. International Journal of Psychology and Psychological Therapy, 14, 85-101.

MARINO, L. (2012). Construct validity of animal-assisted therapy and activities. How important is the animal on AAT? Anthrozoos, 25, s129-s151. DOI: 10.27 52/175303712X13353430377219

Moody, W.J., King, R. y O'Rourke, S. (2002). Attitudes of paediatric medical Ward staff to a dog visitation programme. Journal of Clinical Nursing, 11, 537544. DOI: 10.1046/j.1365-2702.2002.00618.x

Nimer. J. y LUNDAHL. B. (2007). Animal-assisted therapy: A meta-analysis. Anthrozoos, 20, 225-238. DOI: 10.2752/089279307X224773

Perea-Mediavilla, M.A., López-Cepero, J., Tejada, A. y Sarasola, J.L. (2014). Intervenciones asistidas por animales y calidad de vida: expectativas en estudiantes universitarios españoles. Escritos de Psicología, 7, 10-18. DOI: 10.5231/psy.writ.2014.1909

Perkins, J., Bartlett, H., Travers, C. y Rand, J. (2008). Dog-assisted therapy for older people with dementia. Australasian Journal of Ageing. 27. 177-182. DOI: 10.1111/j.1741-6612.2008.00317.x

Pet Partners (s.f.) Understanding the differences between AAA and AAT. Recuperado de www.petpartners.org en agosto de 2013.

RISLEY-CURTISS, C. (2010). Social Work Practitioners and the Human-Companion Animal Bond: A National Study. Social Work, 55, 38-46.

SERPELL. J.A. (2010). Animal-assisted interventions in historical perspective. En H.A. Fine (Ed.). Handbook of animal-assisted therapy: Theoretical foundations and guidelines for practice (3. ${ }^{\mathrm{a}}$ ed.) (pp.17-32). U.S.A.: Academic Press.

Souter, M.A. y Miller, M.D. (2007). Do animal-assisted activities effectively treat depression? A meta-analysis. Anthrozoos, 20, 167-180. DOI: 10.2752/175303707X207954

StEED. H.N. y SMITH. B.S. (2002). Animal assisted activities for geriatric patients. Activities. Adaptation and Aging, 27, 49-61. DOI:10.1300/J016v27n01_04

VeldE. B.P. Cipriani. J. y FiSHER. G. (2005). Resident and therapist views of animal-assisted therapy: implications for occupational therapy practice. Australian Occupational Therapy Journal, 52, 43-50. DOI: 10.1111/j.1440-1330.2004.00442.x

Virués-Ortega, J. y Buela-CASAL, G. (2006). Psychophysiological effects of human-animal interaction: theoretical issues and long-term interaction effects. The Journal of Nervous and Mental Disease, 194, 52-57. DOI: 10.1097/01. nmd.0000195354.03653.63

Wilson, C.C. y NetTing, F.E. (2012). The status of instrument development in the human-animal interaction field. Anthrozoos, 25, S11-S55. DOI: 10.2752/1 $75303712 \times 13353430376977$ 
ANEXO

Cuestionario de Actitudes hacia las Intervenciones Asistidas por Perros (CAINTAP)

A continuación, se presenta una serie de situaciones que pueden darse a la hora de introducir uno de los perros de nuestra asociación en un centro (recurso, asociación ) como en el que está usted trabajando (o le gustaría trabajar). Por favor, indique hasta qué punto está de acuerdo con las siguientes afirmaciones. Recuerde, las respuestas están comprendidas entre muy en desacuerdo (a la izquierda) y muy de acuerdo (a la derecha).

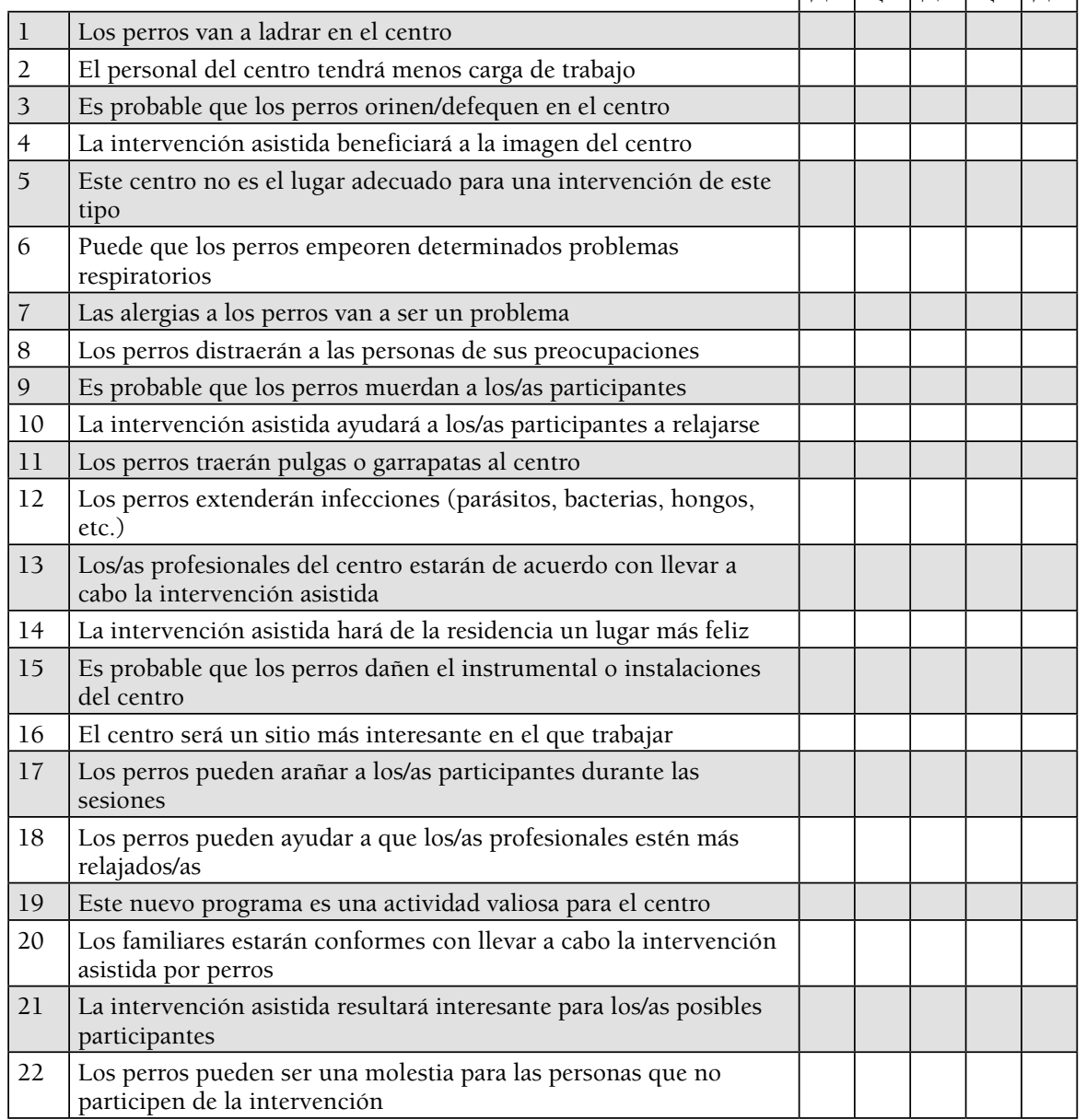

